\title{
Spotlight on risankizumab and its potential in the treatment of plaque psoriasis: evidence to date
}

This article was published in the following Dove Press journal:

Psoriasis: Targets and Therapy

\author{
Álvaro Machado' \\ Tiago Torres ${ }^{1,2}$ \\ 'Department of Dermatology, Centro \\ Hospitalar Universitário do Porto, \\ Porto, Portugal; ' ${ }^{2}$ Instituto de Ciências \\ Biomédicas Abel Salazar, University of \\ Porto, Porto, Portugal
}

\begin{abstract}
Psoriasis is a common chronic immune-mediated skin disease, with systemic involvement and significant impact in patients' quality of life. Several highly specific treatments have been developed over the years, such as tumor necrosis factor- $\alpha$ inhibitors, a nonselective IL-23 inhibitor (ustekinumab), and most recently IL-17 inhibitors. Risankizumab is a monoclonal antibody which targets IL-23p19 without binding IL-12. This novel therapeutic approach is expected to have advantages over the recently approved anti-IL-17 agents, such as the avoidance of Candida infections and neutropenia. In addition, unlike ustekinumab, the selective inhibition of IL-23 may preserve IL-12-dependent functions such as protection against infections and tumor immune surveillance. Risankizumab showed an excellent efficacy when compared to placebo and ustekinumab, with higher Psoriasis Area Severity Index (PASI) 75, PASI 90, and PASI 100 rates, along with a convenient every 12-week maintenance dosing regimen. Overall, risankizumab was well tolerated and the most common adverse event was upper respiratory tract infection. In the near future, further data will be available not only in psoriasis but also in Crohn's disease and psoriatic arthritis fields. Head-to-head trials comparing risankizumab with other IL-23 inhibitors and with IL-17 inhibitors will be crucial to reveal the role of risankizumab in the treatment of psoriasis.
\end{abstract}

Keywords: psoriasis, IL-23, risankizumab

\section{Introduction}

Psoriasis is a common chronic immune-mediated skin disease, with systemic involvement and significant impact in patients' quality of life, ${ }^{1,2}$ with a prevalence ranging between $1.5 \%$ and $5.0 \%$ in most developed countries. ${ }^{2}$

Similar to several immune-mediated inflammatory disorders, psoriasis treatment has evolved immensely. Although conventional treatment is still useful, and sometimes enough for some patients, a great proportion of patients will need other treatment options, with expected better efficacy and, ultimately, an improvement in the quality of life. Over the past decades, our understanding of psoriasis pathogenesis has significantly expanded, allowing the development of highly specific agents, the so-called biologics. ${ }^{3,4}$ Tumor necrosis factor alpha (TNF- $\alpha$ ) inhibitors were probably the first major advance in this regard, as significantly higher clinical responses were achieved. 5 However, some patients did not respond to such agents, motivating further investigation of additional and possibly more crucial targets. Later, ustekinumab, an IL-12/23 inhibitor that selectively targets the common p40 subunit
Correspondence: Tiago Torres

Rua D. Manuel II, s/n, Serviço de Dermatologia Centro Hospitalar Universitário do Porto, piso I, 4050-344 Porto, Portugal

Tel +35I 226097429

Email torres.tiago@outlook.com 
of these cytokines, was developed and not only showed promising clinical responses ${ }^{7,8}$ but also proved to be more effective than TNF- $\alpha$ inhibitors. ${ }^{9}$ Subsequently, as IL-17 was increasingly becoming a recognized key mediator in the pathogenesis of psoriasis, new drugs were developed targeting both IL-17 and its receptor (Figure 1). ${ }^{10}$ These agents have been approved to treat moderate to severe psoriasis ${ }^{11-13}$ and have been shown to have better clinical responses than TNF- $\alpha$ inhibitors ${ }^{14,15}$ and ustekinumab. ${ }^{12,16,17}$ In fact, these agents introduced a paradigm shift in the management of psoriasis, where completely or almost completely clear skin has become the new therapeutic goal. ${ }^{18}$ However, there are some class safety concerns. IL-17 inhibitors are associated with higher risk of mucocutaneous candidiasis and neutropenia, reflecting the role of IL-17 in mucocutaneous protection against extracellular organisms such as Candida albicans and in the granulopoiesis, survival, and activation of neutrophils. Nevertheless, these adverse events (AEs) tend to be easily manageable and usually do not restrict the maintenance of therapy. ${ }^{19,20}$

Most recently, a new class of highly specific drugs is being studied for the treatment of psoriasis - IL-23 inhibitors. Risankizumab is a humanized IgG1 monoclonal antibody which selectively targets the unique p19 subunit of human IL-23. ${ }^{21,22}$ Indeed, this agent seems to have a similar or higher efficacy than IL-17 inhibitors, without the safety concerns mentioned above.
This article aims to review the current knowledge on risankizumab for the treatment of psoriasis.

\section{Advantages of selective IL-23 inhibition}

Nowadays, it is widely accepted that IL-23/IL-17 axis has a crucial role in psoriasis, as in several other immune-mediated diseases. ${ }^{23-29}$ In fact, IL-23 is an essential cytokine for the differentiation, maintenance, and activity of several immune cells, such as T-helper 17 (Th17) and Th22 cells, and is able to induce a self-amplificatory loop by promoting the expression of additional IL-23 receptors. ${ }^{24,27-29}$ The first compound designed to modulate IL-23/IL-17 axis was ustekinumab - an inhibitor of p40 subunit which is shared by two cytokines, IL-23 and IL-12. ${ }^{30}$ Although it proved to be effective, ${ }^{7,8}$ evidence has suggested a more prominent role of IL-23, when comparing to IL-12. Indeed, a predisposition to psoriasis has been linked with genes of the IL-23/Th17 axis, but not with those of the IL-12/Th1 pathway. ${ }^{31}$ In addition, inhibition of IL-12 may have a negative effect in psoriasis. ${ }^{32}$ Using the imiquimod murine model, IL-12 has been shown to play a regulatory role by restraining the infiltration of IL-17Aproducing $\gamma \delta \mathrm{T}$ cells and inducting a protective transcriptional program in keratinocytes, thus limiting skin inflammation. ${ }^{33}$ Furthermore, IL-12 has an anti-inflammatory effect through induction of expression of IL-10 by Th1 cells, in which interferon- $\gamma$ is a key mediator. ${ }^{34}$ Importantly, IL-12 has been

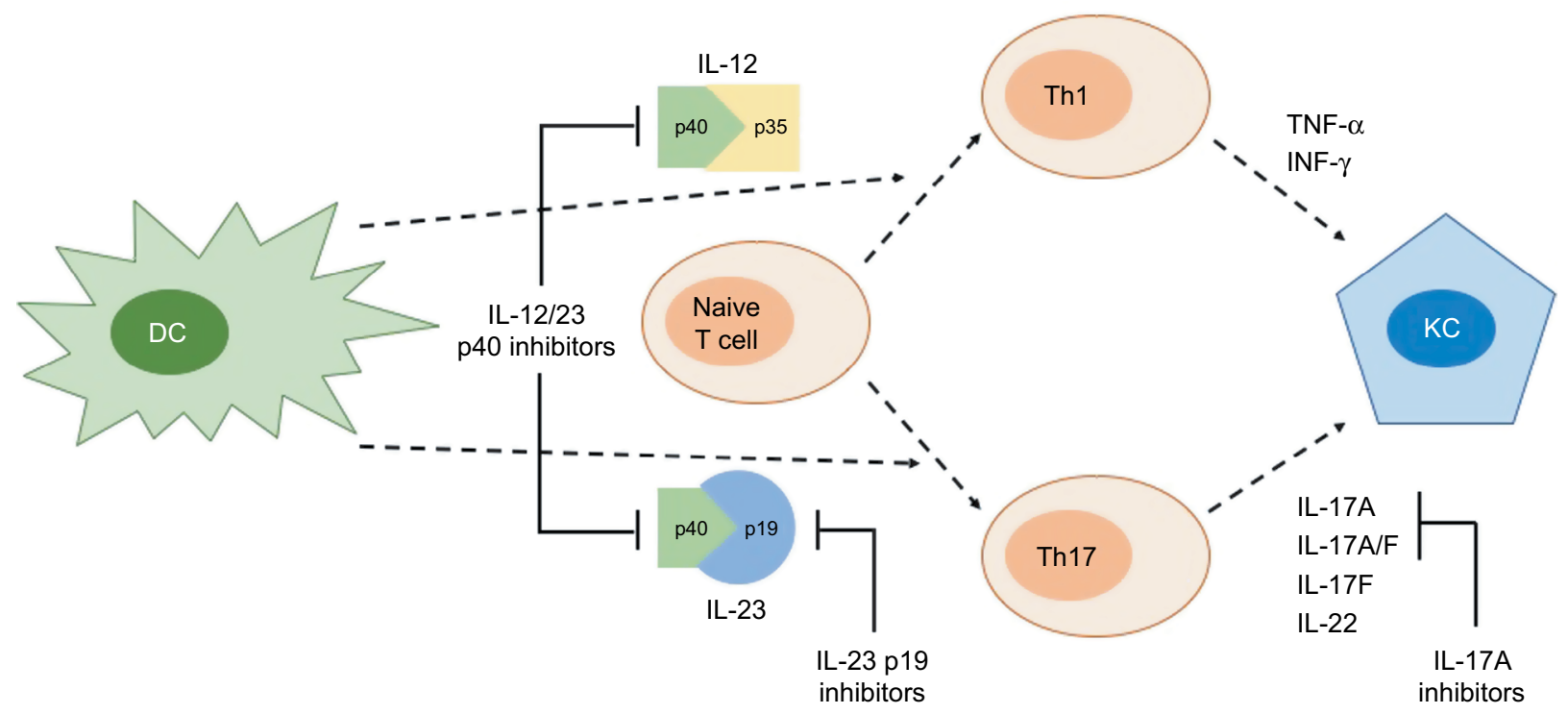

Figure I Summarized schematic differences between IL-I2/23-p40, IL-23p I9, and IL- I7 blockade.

Notes: IL-12/23p40 inhibitors, such as ustekinumab, block IL- 12 and IL-23 common subunit p40, inhibiting both IL-23/Th I7 and IL- I2/Th I pathways; IL-23p I 9 inhibitors, such as risankizumab, guselkumab, and tildrakizumab, selectively block the IL-23/TI7 pathway, avoiding effects on the IL-I2/ThI axis; IL-I7A inhibitors, such as secukinumab and ixekizumab, also target the IL-23/IL-I7 axis, although through downstream blockade of this pathway.

Abbreviations: DC, dendritic cell; INF- $\gamma$, interferon gamma; KC, keratinocyte; ThI and ThI7, T-helper cell I and I7; TNF- $\alpha$, tumor necrosis factor alpha. 
implicated in the protection against bacterial and viral infections $^{35}$ and may play a role in tumor immune surveillance. ${ }^{36,37}$

\section{Risankizumab}

Risankizumab is a humanized IgG1 monoclonal antibody which selectively targets the unique p19 subunit of human IL-23 without binding IL-12. . $^{21} 22$

\section{Pharmacokinetics}

In a Phase I study, ${ }^{21}$ after a single intravenous dose of risankizumab, the geometric mean area under the curve (AUC) $)_{0 \text {-inf }}$ ranged from 2.93 to $1,650 \mathrm{~d} \times \mu \mathrm{g} / \mathrm{mL}$, while maximum serum concentration ranged from 0.311 to $110 \mu \mathrm{g} / \mathrm{mL}$, increasing proportionally with dose. Group mean clearance and terminal phase volume of distribution were $0.33 \mathrm{~L} / \mathrm{d}$ and $10.8 \mathrm{~L}$, respectively. Pharmacokinetic parameter variability was less than $50 \%$. The half-life ranged from 20 to 28 days, while maximal exposures were reached between days 4 and 10 . Subcutaneous bioavailability was $59 \%$ (ratio of $\mathrm{AUC}_{0 \text {-inf }}$ after subcutaneous and intravenous administration). Additionally, investigators also stated that the exposure efficacy was comparable for intravenous and subcutaneous administrations, with maximum PASI responses achievement for doses of $0.25 \mathrm{mg} / \mathrm{kg}$ or greater.

A recent study estimated a clearance, steady-state volume of distribution, and terminal-phase elimination half-life to be $\sim 0.35 \mathrm{~L} /$ day, $11.7 \mathrm{~L}$, and 27 days, respectively, for a typical 90-kg subject with psoriasis with an albumin level of $42 \mathrm{~g} / \mathrm{L}$. Risankizumab absolute subcutaneous bioavailability and absorption rate constant were $72 \%$ and 0.18 day $^{-1}$, respectively. Interindividual variability for clearance was $37 \%$. $^{38}$

\section{Clinical efficacy in psoriasis}

\section{Phase I studies}

The same Phase I study by Krueger et al also showed efficacy outcomes. At week 12, PASI 75 was achieved in $87 \%$, PASI 90 in $58 \%$, and PASI 100 in $16 \%$ of the patients, whereas none of the patients in the placebo group reached any of these clinical responses. At week 24, PASI 75 was achieved in $71 \%$, PASI 90 in $48 \%$, and PASI 100 in $29 \%$ of the patients, compared to $13 \%$ for PASI 75 and $0 \%$ for both PASI 90 and 100 in the placebo group. Additionally, for doses of $0.25 \mathrm{mg} / \mathrm{kg}$ or greater given intravenously or subcutaneously, PASI 75 was achieved in 84\%, PASI 90 in $60 \%$, and PASI 100 in $36 \%$, while among patients receiving risankizumab subcutaneously, the corresponding PASI responses were $100 \%, 85 \%$, and $54 \%$, respectively. Interestingly, PASI improvements were observed as early as week 2 . Regarding the Static Physician Global Assessment (sPGA) evaluation, results were consistent with PASI scores, as all patients getting risankizumab subcutaneously became "clear" or "almost clear" at weeks 12 and 24, compared to none of patients receiving placebo. ${ }^{21}$

\section{Phase II studies}

A Phase II, dose-ranging, multicenter, randomized trial by Papp et al included 166 patients with moderate to severe psoriasis and compared risankizumab with ustekinumab. Patients were randomized to receive risankizumab subcutaneously (18-mg single dose or 90- or 180-mg doses at weeks 0,4 , and 16) or ustekinumab (45 or $90 \mathrm{mg}$, according to body weight, at weeks 0,4 , and 16). The primary endpoint was a PASI 90 or greater response at week 12. Risankizumab was superior to ustekinumab, as $77 \%$ of the patients (90 and 180 mg pooled) achieved the primary endpoint, compared to $40 \%$ in ustekinumab-treated patients. Additionally, at week 12, $63 \%, 98 \%$, and $88 \%$ of the patients achieved PASI 75 with 18-, 90-, and 180-mg doses of risankizumab, respectively, compared to $72 \%$ in ustekinumab-treated patients. With regard to PASI 100, responses of $14 \%, 41 \%$, and $48 \%$ were observed in the 18-, 90-, and 180-mg risankizumab groups, respectively, compared to $18 \%$ in the ustekinumab group. sPGA scores of 0 or 1 were observed in $58 \%, 90 \%$, and $88 \%$ of patients in the $18-, 90-$, and $180-\mathrm{mg}$ risankizumab groups, respectively, compared to $62 \%$ in the ustekinumab group. Furthermore, at week 24, the proportion of patients who achieved a PASI 75 response was 53\%, 90\%, and $88 \%$ for 18-, 90-, and 180-mg dosing regimens of risankizumab, respectively, compared with $70 \%$ in the ustekinumab group. Additionally, at week 24, PASI 90 responses were also assessed, with $28 \%, 63 \%$, and $81 \%$ of risankizumab-treated patients $(18,90$, and $180 \mathrm{mg}$, respectively), compared to $55 \%$ in the ustekinumab group. Interestingly, improvements in PASI score were observed as early as week 2. Clinical responses were generally maintained for up to 20 weeks after the final dose of risankizumab (week 36), in contrast to reduction in clinical responses observed in ustekinumabtreated patients from week 24. Also, complete clearing was maintained in $29 \%$ and $26 \%$ (90 and $180 \mathrm{mg}$, respectively) for up to 32 weeks after the final administration of risankizumab, whereas no patients had complete clearing at 48 weeks in the ustekinumab group. At week 12, 72\% of patients treated with 90 or $180 \mathrm{mg}$ of risankizumab achieved 
a Dermatology Life Quality Index (DLQI) score of 0 or 1, compared to $53 \%$ in the ustekinumab group. Moreover, the percentages of patients with DLQI score of 0 or 1 were correlated with decreases in PASI and sPGA scores (Table 1). ${ }^{22}$

In this study, investigators also assessed scalp, fingernail, and palmoplantar disease. At week 12, a mean reduction in the Psoriasis Scalp Severity Index of $90 \%$ and 94\% (for 90- and 180-mg risankizumab groups, respectively) were observed and sustained over time, compared to $82 \%$ in the ustekinumab group, with a nonsustained response over time. Although fingernail responses were slower, a mean reduction in the Nail Psoriasis Severity Index (NAPSI) score of 40\% was observed at week 12 (for both 90 - and 180-mg risankizumab groups), increasing to $61 \%$ and $73 \%$ for $90-$ and $180-\mathrm{mg}$ groups, respectively, at week 48 , compared to $18 \%$ decrease in NAPSI score in the ustekinumab group. ${ }^{22}$

Finally, skin biopsy samples were obtained from 60 patients. Of which, $54 \%$ and $69 \%$ of the patients in the 90- and 180-mg risankizumab groups, respectively, had global histopathologic assessment graded as "excellent improvement," compared to $29 \%$ in the ustekinumab group. In addition, patients treated with risankizumab, but not with ustekinumab, also had decreased expression of IL-23/IL-17 axis-related genes. ${ }^{22}$

\section{Phase III studies}

Recently, two Phase III studies have been published UltIMMa-1 and UltIMMa-2 - and assessed the efficacy and safety of risankizumab compared to placebo or ustekinumab in patients with moderate to severe plaque psoriasis. ${ }^{39}$ These two studies were replicate Phase III multicenter, randomized, double-blind, placebo-controlled, and active comparatorcontrolled trials. Patients were randomly assigned $(3: 1: 1$; 506 in UltIMMa-1 and 491 in UltIMMa-2) to receive risankizumab, ustekinumab, or placebo, and randomization was stratified by weight ( $\leq 100 \mathrm{~kg}$ vs $>100 \mathrm{~kg}$ ) and previous exposure to TNF inhibitor (yes vs no). In part A of the studies (16-week treatment period), patients received either $150 \mathrm{mg}$ risankizumab, ustekinumab based on weight per label (45 mg for patients with body weight $\leq 100 \mathrm{~kg}$ or $90 \mathrm{mg}$ for patients with body weight $>100 \mathrm{~kg}$ ), or placebo subcutaneously at weeks 0 and 4. In part B (weeks 16-52), patients originally allocated to placebo were switched to $150 \mathrm{mg}$ risankizumab at week 16; during this phase, patients received the study drug subcutaneously at weeks 16,28 , and 40 . Efficacy outcomes were assessed at weeks 4, 8, 12, 16, 22, 28, 34, 40, 46, and 52 , and safety was evaluated throughout the study. The co- primary endpoints were the proportions of patients achieving PASI 90 and sPGA 0 or 1 at week $16 .^{39}$

In both studies, significantly greater proportions of patients receiving risankizumab achieved PASI 90 and sPGA 0 or 1 at week $16(P<0.0001$ vs placebo). At week 16, PASI 90 was achieved by $75.3 \%$ of patients receiving risankizumab compared to $42.0 \%$ receiving ustekinumab and $4.9 \%$ receiving placebo in UltIMMa- 1 , and by $74.8 \%$ of patients receiving risankizumab compared to $47.5 \%$ receiving ustekinumab and $2.0 \%$ receiving placebo in UltIMMa-2 $(P<0.0001$ vs placebo and ustekinumab for both studies). sPGA 0 or 1 was achieved by $87.8 \%$ of risankizumab-treated patients compared to $63.0 \%$ of ustekinumab-treated patients and $7.8 \%$ of placebo-treated patients in UltIMMa-1, and by $83.7 \%$ risankizumab-treated patients compared to $61.6 \%$ of ustekinumab-treated patients and $5.1 \%$ of placebotreated patients in UltIMMa-2 $(P<0.0001$ vs placebo and ustekinumab for both studies). ${ }^{39}$

With regard to complete clearance of psoriatic lesions, PASI 100 was achieved by $35.9 \%$ of risankizumab-treated patients compared to $12.0 \%$ of ustekinumab-treated patients and none of placebo-treated patients in UltIMMa-1, and by $50.7 \%$ of patients receiving risankizumab compared to $24.2 \%$ of patients receiving ustekinumab and $2.0 \%$ of patients receiving placebo in UltIMMa-2 $(P<0.0001$ vs placebo and ustekinumab for both studies). Furthermore, sPGA 0 was achieved by $36.8 \%$ of risankizumab-treated patients compared to $14.0 \%$ of ustekinumab-treated patients and $2.0 \%$ placebo-treated patients in UltIMMa-1, and by $51.0 \%$ of patients receiving risankizumab compared to $25.3 \%$ of patients receiving ustekinumab and $3.1 \%$ of patients receiving placebo in UltIMMa-2 $(P<0.0001$ vs placebo and ustekinumab for both studies). ${ }^{39}$

Interestingly, significantly higher PASI 90 responses were observed starting at week 4 in patients receiving risankizumab compared to placebo (UltIMMa-1: $P=0.0001$; UltIMMa-2: $P<0.0001)$, and at week 8 compared to ustekinumab (UltIMMa-1 and UltIMMa-2: $P<0.0001)$. With regard to sPGA, significantly higher proportions of patients treated with risankizumab achieved sPGA 0 or 1 , starting at week 4 , compared to ustekinumab (UltIMMa-1: $P=0.0047$; UltIMMa-2: $P=0.0129$ ) and placebo (UltIMMa-1 and UltIMMa-2: $P<0.0001$ ). Also, significantly greater proportions of risankizumab-treated patients achieved PASI 75 responses at weeks 12 and 16 compared to ustekinumab (UltIMMa-1: week 12, $P=0.0005$; week 16, $P=0.0034$; UltIMMa-2: $P<0.0001)$ and placebo $(P<0.0001) .{ }^{39}$ 
Additionally, with regard to patient-reported outcomes, the proportion of risankizumab-treated patients who achieved DLQI 0 or 1 and Psoriasis Symptom Scale (PSS) 0 at week 16 was significantly higher compared to both ustekinumab and placebo groups. In UltIMMa-1, $65.8 \%$ of patients receiving risankizumab achieved DLQI 0 or 1 compared to ustekinumab $(43.0 \% ; P<0.0001)$ and placebo $(7.8 \%$; $P<0.0001$ ); in UltIMMa-2, DLQI 0 or 1 was achieved by $66.7 \%$ of patients receiving risankizumab compared to $46.5 \%$ of those receiving ustekinumab $(P=0.0004)$ and $4.1 \%$ of those receiving placebo $(P<0.0001)$. With regard to PSS, at week 16 , PSS 0 was achieved by $29.3 \%$ of patients receiving risankizumab compared to $15.0 \%$ of those receiving ustekinumab $(P=0.0010)$ and $2.0 \%$ of those receiving placebo $(P<0.0001)$ in UltIMMa-1, and by $31.3 \%$ of risankizumabtreated patients compared to $15.2 \%$ of ustekinumab-treated patients $(P=0.0003)$ and none of placebo-treated patients $(P<0.0001)$ in UltIMMa-2. ${ }^{39}$

After week 16, the proportions of patients achieving PASI and sPGA responses continued to increase in patients initially assigned to risankizumab. At week 52, PASI 90 was achieved by $81.9 \%$ of patients on continuous risankizumab compared to the ustekinumab group (44.0\%) in UltIMMa-1, and by $80.6 \%$ of patients on continuous risankizumab compared to the ustekinumab group (50.5\%) in UltIMMa-2 $(P<0.0001$ vs ustekinumab for both studies). At week 52, PASI 100 was achieved by $56.3 \%$ of patients on continuous risankizumab compared to the ustekinumab group (21.0\%) in UltIMMa-1, and by $59.5 \%$ of patients on continuous risankizumab compared to the ustekinumab group (30.3\%) in UltIMMa-2 $(P<0.0001$ for both studies). sPGA 0 at week 52 was achieved by $57.6 \%$ of patients on continuous risankizumab compared to the ustekinumab group (21.0\%) in UltIMMa-1, and by $59.5 \%$ of patients on continuous risankizumab compared to the ustekinumab group (30.3\%) in UltIMMa-2 ( $P<0.0001$ for both studies). Patients initially assigned to placebo switching to risankizumab at week 16 had similar response rates for PASI, sPGA, DLQI, and PSS at week 52 than those initially assigned to risankizumab. Finally, among patients who achieved PASI 90 at week 16 in both studies, a significantly higher proportion of patients continuing risankizumab maintained PASI 90 response through week 52 compared to the ustekinumab group. At week $52,88.4 \%$ of patients on continuous risankizumab maintained PASI 90 response compared to $73.3 \%$ of patients on ustekinumab $(P=0.0009)$ (Table 1). ${ }^{39}$

The final results of other Phase III studies are awaited, comparing risankizumab to adalimumab (IMMvent study), secukinumab (NCT03478787), methotrexate (NCT03219437), and dimethyl fumarate (NCT03255382); assessing the safety and efficacy of risankizumab compared to placebo with withdrawal and retreatment (IMMhance study, NCT02672852); and assessing the safety and efficacy of risankizumab for maintenance in moderate to severe psoriasis (LIMMITLESS study, NCT03047395).

Recently, preliminary data from IMMvent (NCT02694523) were presented. This was a Phase III randomized, doubleblind, active-controlled study evaluating the efficacy and safety of risankizumab compared with adalimumab in adult patients with moderate to severe plaque psoriasis. Patients were randomized $1: 1$ to receive $150 \mathrm{mg}$ risankizumab (weeks $0,4,16$, and 28) or adalimumab (80 $\mathrm{mg}$ at week $0,40 \mathrm{mg}$ every other week from week 1). Adalimumabtreated patients achieving PASI 50 to $<$ PASI 90 at week 16 were rerandomized $1: 1$ to either continue adalimumab or switch to risankizumab (weeks 16, 20, and 32). At week 16, risankizumab-treated patients achieved significantly higher PASI 75 (90.7\% vs $71.7 \%$ ), PASI 90 ( $72.4 \%$ vs $47.4 \%$ ), PASI $100(39.9 \%$ vs $23.0 \%)$, and sPGA $0 / 1(83.7 \%$ vs $60.2 \%)$ response rates compared with adalimumab-treated patients. Additionally, among adalimumab-treated patients with PASI 50 to $<$ PASI 90 responses at week 16 , a significantly higher proportion of patients switching to risankizumab achieved PASI 90 (66.0\% vs $21.4 \%)$ and PASI 100 (39.6\% vs 7.1\%) responses at week 44 compared with patients continuing on adalimumab. Also, PASI 90 and PASI 100 responses were significantly higher in patients receiving risankizumab starting at week 8 compared with adalimumab. Finally, among adalimumab-treated patients achieving PASI 50 to $<$ PASI 90 at week 16, PASI 90 and PASI 100 responses were significantly higher in patients switching to risankizumab, starting at week 20, compared with patients continuing on adalimumab. ${ }^{40}$

Interestingly, a recently presented integrated analysis from Phase III studies UltIMMa-1, UltIMMa-2, and IMMhance revealed that the treatment with risankizumab was associated with superior efficacy compared with placebo regardless of psoriasis treatment history, including prior biologic failure. ${ }^{41}$

\section{Safety and tolerability}

The Phase I study by Krueger et al reported similar rates $(65 \%)$ of side effects in both treatment and placebo groups. The most common AEs were mild to moderate nasopharyngitis, mild to moderate upper respiratory infections, and mild to moderate headache. Importantly, none of 
Table I Summary of key results from clinical trials of risankizumab

\begin{tabular}{|c|c|c|c|c|}
\hline \multirow[t]{2}{*}{ Clinical trial } & \multirow[t]{2}{*}{ Dosing regimen } & \multicolumn{3}{|c|}{ Proportion of patients achieving } \\
\hline & & PASI 50 & PASI 75 & PASI 90 \\
\hline $\begin{array}{l}\text { Phase Il study } \\
\text { (Papp et } \mathrm{al}^{22} \text { ) }\end{array}$ & $\begin{array}{l}\text { 18-mg single dose of } \\
\text { risankizumab at week } 0 \text {; or } \\
90-\mathrm{mg} \text { or } 180-\mathrm{mg} \text { doses of } \\
\text { risankizumab or } 45-\mathrm{mg} \text { or } \\
90-\mathrm{mg} \text { doses of ustekinumab } \\
\text { based on weight }(\leq 100 \mathrm{~kg} \text { vs } \\
>100 \mathrm{~kg}) \text {, at weeks } 0,4 \text {, and } 16\end{array}$ & $\begin{array}{l}\text { At week I2 } \\
\text { Risankizumab: } \\
\text { I8-mg: } 86.0 \% \\
\text { 90-mg: } 100.0 \% \\
(P<0.00 \text { I) } \\
\text { I80-mg: } 92.9 \% \\
\text { Ustekinumab: } 82.5 \%\end{array}$ & $\begin{array}{l}\text { At week I2 } \\
\text { Risankizumab: } \\
\text { I8-mg: } 62.8 \% \\
\text { 90-mg: } 97.6 \% \\
(P<0.00 \text { I) } \\
\text { I80-mg: } 88.1 \% \\
\text { Ustekinumab: } 72.5 \% \\
\text { At week } 24 \\
\text { Risankizumab: } \\
\text { I8-mg: 53.0\% } \\
\text { 90-mg: } 90.0 \% \\
\text { I80-mg: } 88.0 \% \\
\text { Ustekinumab: } 70.0 \%\end{array}$ & $\begin{array}{l}\text { At week I2 } \\
\text { Risankizumab: } \\
\text { I8-mg: } 30.2 \% \\
90-\mathrm{mg}: 73.2 \% \\
(P<0.00 \text { I) } \\
\text { I80-mg: } 78.6 \% \\
(P<0.00 \text { I) } \\
\text { Ustekinumab: } 40.0 \% \\
\text { At week } 24 \\
\text { Risankizumab: } \\
\text { I8-mg: } 28.0 \% \\
90-\mathrm{mg}: 63.0 \% \\
\text { I80-mg: } 81.0 \% \\
\text { Ustekinumab: } 55.0 \%\end{array}$ \\
\hline UltIMMa-I & $\begin{array}{l}\text { Part A: } \\
\text { I50-mg dose of risankizumab, } \\
45-\mathrm{mg} \text { or } 90-\mathrm{mg} \text { doses of } \\
\text { ustekinumab based on weight } \\
\text { ( } \leq 100 \mathrm{~kg} \text { vs }>100 \mathrm{~kg} \text { ), or } \\
\text { placebo, at weeks } 0 \text { and } 4 \\
\text { Part B: } \\
\text { Patients initially assigned to } \\
\text { placebo switched to } 150 \mathrm{mg} \\
\text { risankizumab at week } 16\end{array}$ & Not reported & $\begin{array}{l}\text { At week I2 } \\
\text { Risankizumab: } 86.8 \% \\
\text { Ustekinumab: } 70.0 \% \\
(P=0.0005) \\
\text { Placebo: } 9.8 \% \\
(P<0.000 \text { I) } \\
\text { Placebo to risankizumab: NA }\end{array}$ & $\begin{array}{l}\text { At week I6 } \\
\text { Risankizumab: } 75.3 \% \\
\text { Ustekinumab: } 42.0 \% \\
(P<0.000 \text { I) } \\
\text { Placebo: } 4.9 \% \\
(P<0.000 \text { I) } \\
\text { Placebo to risankizumab: NA } \\
\text { At week } 52 \\
\text { Risankizumab: } 81.9 \% \\
\text { Ustekinumab: } 44.0 \% \\
\text { ( } P<0.000 \text { I) } \\
\text { Placebo: NA } \\
\text { Placebo to risankizumab: } 78.4 \%\end{array}$ \\
\hline UltIMMa-2 & & Not reported & $\begin{array}{l}\text { At week I2 } \\
\text { Risankizumab: } 88.8 \% \\
\text { Ustekinumab: } 69.7 \% \\
(P<0.000 \text { I }) \\
\text { Placebo: } 8.2 \% \\
(P<0.000 \text { I }) \\
\text { Placebo to risankizumab: NA }\end{array}$ & $\begin{array}{l}\text { At week I6 } \\
\text { Risankizumab: } 74.8 \% \\
\text { Ustekinumab: } 47.5 \% \\
(P<0.000 \text { I) } \\
\text { Placebo: } 2.0 \% \\
(P<0.000 \text { I) } \\
\text { Placebo to risankizumab: NA } \\
\text { At week } 52 \\
\text { Risankizumab: } 80.6 \% \\
\text { Ustekinumab: } 50.5 \% \\
\text { ( } P<0.000 \text { I) } \\
\text { Placebo: NA } \\
\text { Placebo to risankizumab: } 85.1 \%\end{array}$ \\
\hline
\end{tabular}

Note: Nonresponder imputation was used to assess binary endpoints missing data. Abbreviation: NA, not applicable.

the serious adverse events (SAEs) verified were considered related to risankizumab treatment by investigators. ${ }^{21}$

The Phase II by Papp et al reported AE rates of $81 \%, 80 \%$, and $69 \%$ for the $18-\mathrm{mg}, 90-\mathrm{mg}$, and $180-\mathrm{mg}$ risankizumab groups, respectively, and $72 \%$ for the ustekinumab group. The most common $\mathrm{AE}$ in all groups was nasopharyngitis. In the 18-mg, 90-mg, and 180-mg risankizumab groups, 5 (12\%), 6 (15\%), and 0 patient, respectively, had SAEs, including two patients with basal cell carcinoma, one patient with myocar- dial infarction, and one patient who had a cerebrovascular accident on the day after elective surgery for preexisting cerebral aneurysm. ${ }^{22}$

In the Phase III studies UltIMMa-1 and UltIMMa-2, the AE rates were similar between groups throughout the study duration in both studies. The most frequent AEs in part A in both studies were viral upper respiratory tract infection, upper respiratory tract infection, psoriasis, and diarrhea. ${ }^{39}$ In part A of UltIMMa-1, AEs occurred in 49.7\% 


\begin{tabular}{|c|c|c|c|}
\hline PASI IO0 & sPGA 0/I & sPGA 0 & PSS 0 \\
\hline $\begin{array}{l}\text { At week I2 } \\
\text { Risankizumab: } \\
\text { I8-mg: I3.9\% } \\
90 \text {-mg: } 41.5 \% \\
(P<0.00 \mathrm{I}) \\
\text { I80-mg: } 47.6 \% \\
(P<0.00 \mathrm{I}) \\
\text { Ustekinumab: } \mid 7.5 \%\end{array}$ & $\begin{array}{l}\text { At week I2 } \\
\text { Risankizumab: } \\
\text { I8-mg: } 58.1 \% \\
90 \text {-mg: } 90.2 \% \\
(P<0.00 \text { I }) \\
\text { I80-mg: } 88.1 \% \\
(P<0.00 \text { I) } \\
\text { Ustekinumab: } 62.5 \%\end{array}$ & Not reported & Not reported \\
\hline $\begin{array}{l}\text { At week I6 } \\
\text { Risankizumab: } 35.9 \% \\
\text { Ustekinumab: } 12.0 \% \\
(P<0.000 \mathrm{I}) \\
\text { Placebo: } 0 \% \\
(P<0.000 \mathrm{I}) \\
\text { Placebo to risankizumab: NA } \\
\text { At week } 52 \\
\text { Risankizumab: } 56.3 \% \\
\text { Ustekinumab: } 21.0 \% \\
(P<0.000 \mathrm{I}) \\
\text { Placebo: NA } \\
\text { Placebo to risankizumab: } 54.6 \%\end{array}$ & $\begin{array}{l}\text { At week I2 } \\
\text { Risankizumab: } 82.2 \% \\
\text { Ustekinumab: } 65.0 \% \\
(P=0.0007) \\
\text { Placebo: } 8.8 \% \\
(P<0.000 \text { I) } \\
\text { Placebo to risankizumab: NA } \\
\text { At week I6 } \\
\text { Risankizumab: } 87.8 \% \\
\text { Ustekinumab: } 63.0 \% \\
(P<0.000 \mathrm{I}) \\
\text { Placebo: } 7.8 \% \\
(P<0.000 \mathrm{I}) \\
\text { Placebo to risankizumab: NA }\end{array}$ & $\begin{array}{l}\text { At week I6 } \\
\text { Risankizumab: } 36.8 \% \\
\text { Ustekinumab: } 14.0 \% \\
(P<0.000 \text { I }) \\
\text { Placebo: } 2.0 \% \\
(P<0.000 \text { I) } \\
\text { Placebo to risankizumab: NA } \\
\text { At week } 52 \\
\text { Risankizumab: } 57.6 \% \\
\text { Ustekinumab: } 21.0 \% \\
(P<0.000 \text { I) } \\
\text { Placebo: NA } \\
\text { Placebo to risankizumab: } 54.6 \%\end{array}$ & $\begin{array}{l}\text { At week I6 } \\
\text { Risankizumab: } 29.3 \% \\
\text { Ustekinumab: } 15.0 \% \\
(P=0.0010) \\
\text { Placebo: } 2.0 \% \\
(P<0.000 \text { I }) \\
\text { Placebo to risankizumab: NA }\end{array}$ \\
\hline $\begin{array}{l}\text { At week I6 } \\
\text { Risankizumab: } 50.7 \% \\
\text { Ustekinumab: } 24.2 \% \\
(P<0.000 \mathrm{I}) \\
\text { Placebo: } 2.0 \% \\
(P<0.000 \mathrm{I}) \\
\text { Placebo to risankizumab: NA } \\
\text { At week } 52 \\
\text { Risankizumab: } 59.5 \% \\
\text { Ustekinumab: } 30.3 \% \\
(P<0.000 \mathrm{I}) \\
\text { Placebo: NA } \\
\text { Placebo to risankizumab: } 67.0 \%\end{array}$ & $\begin{array}{l}\text { At week I2 } \\
\text { Risankizumab: } 82.3 \% \\
\text { Ustekinumab: } 64.6 \% \\
(P=0.0006) \\
\text { Placebo: } 9.2 \% \\
(P<0.000 I) \\
\text { Placebo to risankizumab: NA } \\
\text { At week I6 } \\
\text { Risankizumab: } 83.7 \% \\
\text { Ustekinumab: } 61.6 \% \\
(P<0.000 \mathrm{I}) \\
\text { Placebo: } 5.1 \% \\
(P<0.000 \mathrm{I}) \\
\text { Placebo to risankizumab: NA }\end{array}$ & $\begin{array}{l}\text { At week I6 } \\
\text { Risankizumab: } 51.0 \% \\
\text { Ustekinumab: } 25.3 \% \\
(P<0.000 \text { I }) \\
\text { Placebo: } 3.1 \% \\
(P<0.000 \text { I) } \\
\text { Placebo to risankizumab: NA } \\
\text { At week } 52 \\
\text { Risankizumab: } 59.5 \% \\
\text { Ustekinumab: } 30.3 \% \\
(P<0.000 \text { I) } \\
\text { Placebo: NA } \\
\text { Placebo to risankizumab: } 67.0 \%\end{array}$ & $\begin{array}{l}\text { At week I6 } \\
\text { Risankizumab: } 31.3 \% \\
\text { Ustekinumab: } 15.2 \% \\
(P=0.0003) \\
\text { Placebo: } 0 \% \\
(P<0.000 \mathrm{I}) \\
\text { Placebo to risankizumab: NA }\end{array}$ \\
\hline
\end{tabular}

of the patients on risankizumab, $50.0 \%$ on ustekinumab, and $51.0 \%$ on placebo, and in part A of UltIMMa-2, AEs occurred in $45.6 \%$ of the patients on risankizumab, $53.5 \%$ on ustekinumab, and $45.9 \%$ on placebo. SAEs were reported in $2.3 \%$ of risankizumab-treated patients, $8.0 \%$ of ustekinumab-treated patients, and $2.9 \%$ of placebo-treated patients in UltIMMa-1, and in $2.0 \%$ of risankizumab-treated patients, $3.0 \%$ of ustekinumab-treated patients, and $1.0 \%$ of placebo-treated patients in UltIMMa-2. Serious infections occurred in $0.3 \%$ in the risankizumab group and $3.0 \%$ in the ustekinumab group in UltIMMa-1, and in $1.0 \%$ in both risankizumab and ustekinumab groups in UltIMMa-2. In part $B$, the most frequent AEs were viral upper respiratory tract infection, upper respiratory tract infection, urinary tract infection, influenza, and headache in both studies. In part B of UltIMMa-1, AEs occurred in $61.3 \%$ of the patients continuing on risankizumab, $66.7 \%$ on ustekinumab, and $67.0 \%$ of the patients switching from placebo to risankizumab, while in 
part B of UltIMMa-2, AEs occurred in $55.7 \%$ of the patients continuing on risankizumab, $74.5 \%$ on ustekinumab, and $64.9 \%$ of the patients continuing on risankizumab. SAEs were reported in $5.4 \%$ of risankizumab-treated patients, $4.0 \%$ of ustekinumab-treated patients, and $3.1 \%$ of the patients continuing on risankizumab in UltIMMa-1, and in $4.5 \%$ of risankizumab-treated patients, $4.3 \%$ of ustekinumab-treated patients, and $3.2 \%$ of the patients continuing on risankizumab in UltIMMa-2. During part B, serious infections occurred in $0.7 \%$ of the patients on risankizumab in both UltIMMa-1 and UltIMMa-2, and in $1.0 \%$ of the patients switching from placebo to risankizumab and ustekinumab in UltIMMa-2. In part A, malignancy was reported in two patients on risankizumab (squamous cell carcinoma in UltIMMa-1; basal cell carcinoma in UltIMMa-2) and in one patient in the placebo group (squamous cell carcinoma in UltIMMa-1). Of note, there were no events of tuberculosis, opportunistic infections, major adverse cardiovascular events (MACEs), or serious hypersensitivity across both studies. During part B, two cases of latent tuberculosis were reported in the risankizumab treatment group. Through week 52, two patients on risankizumab had MACEs, including one patient with cardiovascular death and one patient with type 1 myocardial infarction. Malignancy was reported in one patient on continuous risankizumab (basal cell carcinoma in UltIMMa-2), in one patient on ustekinumab (prostate cancer in UltIMMa-2), and in two patients switching from placebo to risankizumab (one patient with both basal cell and squamous cell carcinoma in UltIMMa-1; one patient with breast cancer in UltIMMa-2). ${ }^{39}$

\section{Immunogenicity}

Krueger et al reported anti-drug antibodies in $15 \%$ of the patients, although no association with loss of efficacy was noted. ${ }^{21}$ A similar incidence was reported by Papp et al (14\% of patients); in most patients antibodies were transient and with low titers, or both; also, three patients had neutralizing anti-drug antibodies. ${ }^{22}$

\section{Discussion}

The IL-23/IL-17 axis is currently considered to be essential in the pathogenesis of psoriasis. Laboratory studies have identified this axis as the primary signaling pathway leading to characteristic molecular, cellular, and structural changes in psoriatic skin. ${ }^{3,29,42,43}$ The critical importance of the IL-23/ T17 axis in psoriasis is highlighted by the high efficacy and rapid onset of action seen with IL-17 antagonists and most recently with IL-23 antagonists. ${ }^{29}$ However, anti-IL-23 agents may provide equal or higher clinical efficacy of anti-IL-17 agents, without the AEs of the latter, namely mucocutaneous candidiasis and neutropenia. ${ }^{19,20}$

After the recent approval of guselkumab for the treatment of moderate to severe plaque psoriasis, ${ }^{44}$ risankizumab may be the next agent in the class to enter the market after showing excellent results in Phase II and Phase III trials in comparison to both placebo and ustekinumab, ${ }^{22,39}$ a standard of treatment for most psoriatic patients under biological treatment, while offering a similar every 12 -week maintenance dosing regimen as ustekinumab, known for its high convenience of use. Additionally, risankizumab has shown response rates significantly higher than those seen with TNF- $\alpha^{45}$ and numerically superior than those of IL-17 inhibitors. ${ }^{46,47}$

The selective inhibition of IL-23 may present several advantages compared to the inhibition of $\mathrm{p} 40$ subunit shared by IL-23 and IL-12 and even a downstream inhibition of IL-17 or its receptor. Early clinical studies showed relatively long-term treatment responses in some patients with just a single doses of IL-23p19 inhibition. ${ }^{21,48}$ The explanation behind this impressive clinical response may rely in part by promoting transdifferentiation of $\mathrm{Th} 17$ cells into regulatory $\mathrm{T}$ cell or Th1 populations. ${ }^{49,50}$ Also, risankizumab maintained high FoxP3 mRNA levels in posttreatment lesional skin, indicating that it may increase regulatory T-cell levels or function in resolved psoriatic lesions. ${ }^{21}$ Other cytokines may similarly be affected with the inhibition of p19 subunit, besides IL-23, such as IL-39, a recently discovered proinflammatory cytokine likely expressed in psoriatic skin. ${ }^{43,51}$ Additionally, blockade of IL-23 through the inhibition of the p19 subunit would also allow IL-12 and IL-Y to maintain their beneficial anti-inflammatory effects on T17-centered inflammation in the skin. ${ }^{43}$ Indeed, IL-12 has limited to no role in psoriasis and its inhibition may have a negative effect in psoriasis, as IL-12 has been shown to play a regulatory role by preventing the infiltration of IL-17A-producing $\gamma \delta$ T-cells, therefore limiting skin inflammation. ${ }^{33}$ Notably, IL-12 has been implicated in the protection against intracellular pathogens $s^{5,35,52}$ and may play a role in tumor immune surveillance. ${ }^{36,37}$

Overall, risankizumab showed a similar safety and tolerability profile compared to ustekinumab and placebo. ${ }^{22,39}$ Two major MACEs were reported in the risankizumab treatment group of UltIMMa-2, but both patients had several cardiovascular risk factors and the events were not considered to be related to the study drug. ${ }^{39}$ Additionally, although MACEs 
have been associated with the use of anti-IL-12/23p40 agents (ustekinumab and briakinumab) in patients with psoriasis, the 5-year long-term safety data of ustekinumab and the Psoriasis Longitudinal Assessment and Registry (PSOLAR) data have shown neither increase nor decrease in the rate of MACEs over time, ${ }^{53,54}$ and so far there seems to be no increased risk of MACEs with risankizumab. Nevertheless, future studies with more patients and longer follow-up periods will help establish a more complete safety profile.

Multiple studies comparing risankizumab to adalimumab or secukinumab are ongoing, not only in psoriasis but also in Crohn's disease (NCT03105102, NCT03105128, NCT03104413, NCT02513459) and psoriatic arthritis (NCT02719171, NCT02986373, NCT03671148, NCT03675308). In fact, promising preliminary results were observed regarding the use of risankizumab in Crohn's disease. ${ }^{55-57}$

Altogether, psoriasis, once a difficult-to-treat disease, represents now a success in terms of clinical efficacy due to the highly specific treatments we have nowadays. In fact, the high efficacy of these novel agents has established very high primary study end points used in clinical trials, as PASI 90 is now regarded as the new treatment standard, ${ }^{3,18,29}$ and in some patients, long-term remission of psoriasis activity may be a possibility. However, some psoriatic patients do not improve or just respond partially with IL-17 or IL-23 blockade, highlighting the complexity of the disease and motivating further investigation to fully understand what is behind the skin of our patients.

\section{Conclusion}

Risankizumab is a monoclonal antibody that selectively targets IL-23, offering remarkable rates of complete or near complete clearance of skin disease, along with a good safety profile so far and an excellent dosing regimen. Data from clinical trials comparing risankizumab with other IL-23 and IL-17 inhibitors will be essential to establish the value of risankizumab in the treatment of psoriasis.

\section{Disclosure}

Álvaro Machado has no conflicts of interest in this work. Tiago Torres has participated in clinical trials sponsored by AbbVie, Amgen, Boehringer Ingelheim, and Novartis and has received honoraria for acting as a consultant and/or as a speaker at events sponsored by AbbVie, Amgen, Boehringer Ingelheim, Biogen, Celgene, Janssen, Leo-Pharma, Eli Lilly, MSD, Novartis, Pfizer, Samsung.

\section{References}

1. Rapp SR, Feldman SR, Exum ML, Fleischer AB, Reboussin DM. Psoriasis causes as much disability as other major medical diseases. J Am Acad Dermatol. 1999;41(3 Pt 1):401-407.

2. Parisi R, Symmons DP, Griffiths CE, Ashcroft DM, Identification and Management of Psoriasis and Associated ComorbidiTy (IMPACT) project team. Global epidemiology of psoriasis: a systematic review of incidence and prevalence. J Invest Dermatol. 2013;133(2):377-385.

3. Hawkes JE, Chan TC, Krueger JG. Psoriasis pathogenesis and the development of novel targeted immune therapies. J Allergy Clin Immunol. 2017;140(3):645-653.

4. Torres T. Inhibidores selectivos de la IL-23: los recién llegados al tratamiento de la psoriasis. Actas Dermo-Sifiliográficas. 2018;109(8): 674-676.

5. Torres T. Selective Interleukin- 23 p19 inhibition: another game changer in psoriasis? Focus on risankizumab. Drugs. 2017;77(14):1493-1503.

6. Machado Á, Torres T. Guselkumab for the treatment of psoriasis. BioDrugs. 2018;32(2):119-128.

7. Leonardi CL, Kimball AB, Papp KA, et al. Efficacy and safety of ustekinumab, a human interleukin-12/23 monoclonal antibody, in patients with psoriasis: 76-week results from a randomised, doubleblind, placebo-controlled trial (PHOENIX 1). Lancet. 2008;371(9625): 1665-1674.

8. Papp KA, Langley RG, Lebwohl M, et al. Efficacy and safety of ustekinumab, a human interleukin-12/23 monoclonal antibody, in patients with psoriasis: 52-week results from a randomised, doubleblind, placebo-controlled trial (PHOENIX 2). Lancet. 2008;371(9625): $1675-1684$.

9. Strober BE, Bissonnette R, Fiorentino D, et al. Comparative effectiveness of biologic agents for the treatment of psoriasis in a real-world setting: results from a large, prospective, observational study (Psoriasis Longitudinal Assessment and Registry [PSOLAR]. JAm Acad Dermatol. 2016;74(5):851-861.

10. Kirkham BW, Kavanaugh A, Reich K. Interleukin-17A: a unique pathway in immune-mediated diseases: psoriasis, psoriatic arthritis and rheumatoid arthritis. Immunology. 2014;141(2):133-142.

11. Gordon KB, Blauvelt A, Papp KA, et al. Phase 3 trials of ixekizumab in moderate-to-severe plaque psoriasis. $N$ Engl J Med. 2016;375(4): $345-356$.

12. Thaçi D, Blauvelt A, Reich K, et al. Secukinumab is superior to ustekinumab in clearing skin of subjects with moderate to severe plaque psoriasis: CLEAR, a randomized controlled trial. JAm Acad Dermatol. 2015;73(3):400-409.

13. Puig L. Brodalumab: the first anti-IL-17 receptor agent for psoriasis Drugs Today. 2017;53(5):283.

14. Griffiths CE, Reich K, Lebwohl M, et al. Comparison of ixekizumab with etanercept or placebo in moderate-to-severe psoriasis (UNCOVER-2 and UNCOVER-3): results from two phase 3 randomised trials. Lancet. 2015;386(9993):541-551.

15. Langley RG, Elewski BE, Lebwohl M, et al. Secukinumab in plaque psoriasis-results of two phase 3 trials. NEngl J Med.2014;371(4):326-338.

16. Abraham C, Dulai PS, Vermeire S, Sandborn WJ. Lessons learned from trials targeting cytokine pathways in patients with inflammatory bowel diseases. Gastroenterology. 2017;152(2):374-388.

17. Amin M, Darji K, No DJ, Wu JJ, Dj N, Jj W. Review of phase III trial data on IL-23 inhibitors tildrakizumab and guselkumab for psoriasis. J Eur Acad Dermatol Venereol. 2017;31(10):1627-1632.

18. Torres T, Puig L. Treatment goals for psoriasis: Should PASI 90 become the standard of care? Actas Dermosifiliogr. 2015;106(3):155-157.

19. Saunte DM, Mrowietz U, Puig L, Zachariae C. Candida infections in patients with psoriasis and psoriatic arthritis treated with interleukin-17 inhibitors and their practical management. Br J Dermatol. 2017;177(1):47-62.

20. Blauvelt A. Safety of secukinumab in the treatment of psoriasis. Expert Opin Drug Saf. 2016;15(10):1413-1420. 
21. Krueger JG, Ferris LK, Menter A, et al. Anti-IL-23A mAb BI 655066 for treatment of moderate-to-severe psoriasis: safety, efficacy, pharmacokinetics, and biomarker results of a single-rising-dose, randomized, double-blind, placebo-controlled trial. J Allergy Clin Immunol. 2015;136(1):116-124.

22. Papp KA, Blauvelt A, Bukhalo $M$, et al. Risankizumab versus ustekinumab for moderate-to-severe plaque psoriasis. $N$ Engl J Med. 2017;376(16):1551-1560.

23. Toussirot E. The IL23/Th17 pathway as a therapeutic target in chronic inflammatory diseases. Inflamm Allergy Drug Targets. 2012;11(2):159-168.

24. Girolomoni G, Strohal R, Puig L, et al. The role of IL-23 and the IL-23/ $\mathrm{T}_{\mathrm{H}} 17$ immune axis in the pathogenesis and treatment of psoriasis. $J$ Eur Acad Dermatol Venereol. 2017;31(10):1616-1626.

25. Gaffen SL, Jain R, Garg AV, Cua DJ. The IL-23-IL-17 immune axis: from mechanisms to therapeutic testing. Nat Rev Immunol. 2014;14(9):585-600.

26. Fragoulis GE, Siebert S, Mcinnes IB. Therapeutic targeting of IL-17 and IL-23 cytokines in immune-mediated diseases. Annu Rev Med. 2016;67(1):337-353.

27. Chiricozzi A, Saraceno R, Chimenti MS, Guttman-Yassky E, Krueger JG. Role of IL-23 in the pathogenesis of psoriasis: a novel potential therapeutic target? Expert Opin Ther Targets. 2014;18(5):513-525.

28. Chiricozzi A, Romanelli P, Volpe E, Borsellino G, Romanelli M. Scanning the immunopathogenesis of psoriasis. Int J Mol Sci. 2018;19(1):179.

29. Chan TC, Hawkes JE, Krueger JG. Interleukin 23 in the skin: role in psoriasis pathogenesis and selective interleukin 23 blockade as treatment. Ther Adv Chronic Dis. 2018;9(5):111-119.

30. Cingoz O, Ustekinumab CO. Ustekinumab. MAbs. 2009;1(3):216-221.

31. Levin AA, Gottlieb AB. Specific targeting of interleukin-23p19 as effective treatment for psoriasis. JAm Acad Dermatol. 2014;70(3):555-561.

32. Kulig P, Musiol S, Freiberger SN, et al. IL-12 protects from psoriasiform skin inflammation. Nat Commun. 2016;7:13466.

33. van der Fits L, Mourits S, Voerman JS, et al. Imiquimod-induced psoriasis-like skin inflammation in mice is mediated via the IL-23/ IL-17 axis. J Immunol. 2009;182(9):5836-5845.

34. Chang HD, Radbruch A. The pro- and anti-inflammatory potential of interleukin-12. Ann NY Acad Sci. 2007;1109(30):40-46.

35. Hamza T, Barnett JB, Li B. Interleukin 12 a key immunoregulatory cytokine in infection applications. Int J Mol Sci. 2010;11(3):789-806.

36. Lu X. Impact of IL-12 in Cancer. Curr Cancer Drug Targets. 2017;17(8):682-697.

37. Gately MK, Renzetti LM, Magram J, et al. The interleukin-12/ interleukin-12-receptor system: role in normal and pathologic immune responses. Annu Rev Immunol. 1998;16(1):495-521.

38. Suleiman AA, Khatri A, Minocha M, Othman AA. Population pharmacokinetics of the Interleukin-23 inhibitor risankizumab in subjects with psoriasis and Crohn's disease: analyses of Phase I and II trials. Clin Pharmacokinet. 2018;57(8):977-988.

39. Gordon KB, Strober B, Lebwohl M, et al. Efficacy and safety of risankizumab in moderate-to-severe plaque psoriasis (UltIMMa-1 and UltIMMa-2): results from two double-blind, randomised, placebo-controlled and ustekinumab-controlled phase 3 trials. Lancet. 2018;392(10148):650-661.

40. Reich K, Gooderham M, Thaçi D, Crowley J, Ryan C, Krueger J. Efficacy and Safety of Risankizumab Compared with Adalimumab in Patients with Moderate-to-Severe Plaque Psoriasis: Results from the Phase 3 IMMvent Trial. Paris: In: 27th EADV Congress; 2018.

Psoriasis: Targets and Therapy

\section{Publish your work in this journal}

Psoriasis: Targets and Therapy is international, peer-reviewed, open access journal focusing on psoriasis, nail psoriasis, psoriatic arthritis and related conditions, identification of therapeutic targets and the optimal use of integrated treatment interventions to achieve improved outcomes
41. Strober B, Lambert J, Gu Y, Zhan T, Thompson E, Valdecantos WMA. Impact of Prior Treatment History on Efficacy of Risankizumab Compared with Placebo in Patients with Moderate-to-Severe Plaque Psoriasis: Integrated Analyses from Three Phase 3 Trials. Paris: In: 27th EADV Congress; 2018.

42. Kim J, Krueger JG. Highly effective new treatments for psoriasis target the IL-23/Type $17 \mathrm{~T}$ cell autoimmune axis. Annu Rev Med. 2017;68(1):255-269.

43. Hawkes JE, Yan BY, Chan TC, Krueger JG. Discovery of the IL-23/ IL-17 signaling pathway and the treatment of psoriasis. J Immunol. 2018;201(6):1605-1613.

44. Markham A. Guselkumab: first global approval. Drugs. 2017;77(13): 1487-1492.

45. Signorovitch JE, Betts KA, Yan YS, et al. Comparative efficacy of biological treatments for moderate-to-severe psoriasis: a network metaanalysis adjusting for cross-trial differences in reference arm response. Br J Dermatol. 2015;172(2):504-512.

46. Blauvelt A, Reich K, Tsai TF, et al. Secukinumab is superior to ustekinumab in clearing skin of subjects with moderate-to-severe plaque psoriasis up to 1 year: results from the CLEAR study. $J$ Am Acad Dermatol. 2017;76(1):60-69.

47. Reich K, Pinter A, Lacour JP, et al. Comparison of ixekizumab with ustekinumab in moderate-to-severe psoriasis: 24-week results from IXORA-S, a phase III study. Br J Dermatol. 2017;177(4):1014-1023.

48. Sofen H, Smith S, Matheson RT, et al. Guselkumab (an IL23 -specific $\mathrm{mAb}$ ) demonstrates clinical and molecular response in patients with moderate-to-severe psoriasis. J Allergy Clin Immunol. 2014;133(4):1032-1040.

49. Jäger A, Kuchroo VK, Effector KVK. Effector and regulatory T-cell subsets in autoimmunity and tissue inflammation. Scand J Immunol. 2010;72(3):173-184.

50. Gagliani N, Amezcua Vesely MC, Iseppon A, et al. Th17 cells transdifferentiate into regulatory $\mathrm{T}$ cells during resolution of inflammation. Nature. 2015;523(7559):221-225.

51. Cline A, Feldman SR. Risankizumab for psoriasis. Lancet. 2018;392(10148):616-617.

52. Puig L. The role of IL 23 in the treatment of psoriasis. Expert Rev Clin Immunol. 2017;13(6):525-534.

53. Papp K, Gottlieb AB, Naldi L, et al. Safety Surveillance for Ustekinumab and Other Psoriasis Treatments From the Psoriasis Longitudinal Assessment and Registry (PSOLAR). J Drugs Dermatol. 2015;14(7): 706-714.

54. Papp KA, Griffiths CE, Gordon K, et al. Long-term safety of ustekinumab in patients with moderate-to-severe psoriasis: final results from 5 years of follow-up. Br J Dermatol. 2013;168(4):844-854.

55. Feagan BG, Panés J, Ferrante M. Risankizumab in patients with moderate to severe Crohn's disease: an open-label extension study. Lancet Gastroenterol Hepatol. 2018.

56. Feagan BG, Sandborn WJ, D'Haens G, et al. Induction therapy with the selective interleukin-23 inhibitor risankizumab in patients with moderate-to-severe Crohn's disease: a randomised, doubleblind, placebo-controlled phase 2 study. Lancet. 2017;389(10080): 1699-1709.

57. Visvanathan S, Baum P, Salas A. Selective IL-23 inhibition by risankizumab modulates the molecular profile in the colon and Ileum of patients with active Crohn's disease: results from a randomised Phase II biopsy sub-study. J Crohn's Colitis. 2018;380.

Dovepress

and quality of life. Visit http://www.dovepress.com/testimonials.php to read real quotes from published authors. 